\title{
Hydrodynamics of an inertial active droplet
}

\author{
A. Dhar ${ }^{1}$, P. S. Burada ${ }^{1,2}$ and G. P. Raja Sekhar ${ }^{1,3, \dagger}$ \\ ${ }^{1}$ Centre for Theoretical Studies, Indian Institute of Technology Kharagpur, Kharagpur 721302, India \\ ${ }^{2}$ Department of Physics, Indian Institute of Technology Kharagpur, Kharagpur 721302, India \\ ${ }^{3}$ Department of Mathematics, Indian Institute of Technology Kharagpur, Kharagpur 721302, India
}

(Received 4 January 2020; revised 22 April 2020; accepted 30 July 2020)

\begin{abstract}
Extensive studies have focused on the self-propulsion of a droplet in a viscous environment driven by the Marangoni effect in the absence of inertial effects. In order to capture the influence of inertia on the self-propulsion of a droplet, we use the singular perturbation solution for small but finite Reynolds number $(R e)$ flow past a spherical droplet with inhomogeneous surface tension. We calculate the swimming speed and the corresponding flow fields generated by the droplet in an axisymmetric unbounded medium at $O\left(R e^{2}\right)$. The present results reveal how the choice of the stress parameter $\sigma$, which is the ratio of the first two modes of the induced stress field, distinguishes between the different swimming styles, and determines the role of inertia on the swimming speed, energy expenditure and swimming efficiency of the droplet. Inertia enhances the swimming speed and the associated swimming efficiency of the droplet by abating the energy expenditure. It is striking to observe how a droplet swimmer with $\sigma<0$ has a competitive advantage over a rigid squirmer with an equivalent surface activity due to the existence of an internal flow. We independently treat the potential influence of the viscosity ratio on the swimming properties of the droplet at finite $R e$. Additionally, using linear stability analysis, we provide insights into the stability of the estimated migration velocity at $O(R e)$. We argue that the droplet achieves a distinct stable equilibrium velocity, which occurs due to the inertial effect of the surrounding medium.
\end{abstract}

Key words: drops, swimming/flying

\section{Introduction}

Swimming droplets are artificial micro-swimmers that are driven by an asymmetric distribution of external energy sources across the interface (Maass et al. 2016; Weber et al. 2019). These systems are of tremendous interest as experimental models for mimicking the motion of several biological systems (Čejková et al. 2017; Li et al. 2018). For most motile biological systems, the necessary isotropic symmetry breaking for propulsion occurs intrinsically, i.e. through the beating of the associated appendages cilia or flagella (Lauga \& Powers 2009) or due to shape deformation (D'Ambrosio \& Sinigaglia 2003). However, for droplets, typically the swimmer itself is perfectly

$\dagger$ Email address for correspondence: rajas@iitkgp.ac.in 
isotropic, and the associated symmetry is broken through the external energy sources (Herminghaus et al. 2014; Yoshinaga 2017). In the literature, the propulsion mechanisms of several external sources are believed to be reasonably well understood (Saintillan \& Shelley 2015; Seemann, Fleury \& Maass 2016; Kree, Burada \& Zippelius 2017). Theoretical as well as numerical studies have considered droplets in a chemically reacting fluid (Kitahata et al. 2011; Thutupalli \& Herminghaus 2011; Fadda et al. 2017), in an inhomogeneous temperature field (Subramanian 1992; Karbalaei, Kumar \& Cho 2016) or droplets that move owing to liquid-liquid phase separation (Lamorgese \& Mauri 2011, 2017) etc. In particular, active droplets driven by nonlinear advection effects of the surfactants (Furtado, Pooley \& Yeomans 2008; Mozorov \& Michelin 2019) or the thermal field (Balasubramaniam \& Subramanian 2000; Zhang, Subramanian \& Balasubramaniam 2001) and surfactant-stabilized droplets driven by solubilization (Pena \& Miller 2006; Ariyaprakai \& Dungan 2008) have gained significant attention in the last decade.

All the sources, as mentioned earlier, cause the interface tension of the droplet to vary along the surface. This phenomenon is known as the Marangoni effect (Scriven \& Sternling 1960; Lauga \& Davis 2012). There exist extensive studies for the flow field around a Marangoni-driven active droplet in the quasi-steady Stokesian regime (Schmitt \& Stark 2013, 2016a,b; Masoud \& Stone 2014; Stricker 2017) neglecting the inertial effect. Inertial swimming within the context of biological systems serves as a useful tool in several scientific applications (Reynolds 1965; Hamel et al. 2011). Wu et al. (2009) have demonstrated kinematic separation of mixed bacteria streaming against a wall and shown that the larger bacteria have a faster rate than the smaller bacteria. However, there exist preferably a limited number of studies that have quantified the effects of convective inertia on the kinematics and energetics of swimming droplets (Li \& Mao 2001; Mason \& Moremedi 2011) which are potential artificial analogues of biological systems.

Under the influence of inertial effects due to the external fluid, i.e. at finite Reynolds number $(R e)$, the leading-order approximation to the flow can be found by solving the standard Stokes equation. In an attempt to find the first-order correction due to the Reynolds number, an approximation via a regular perturbation expansion fails, as the computed velocity field invalidates the uniform far-field boundary condition and the higher approximations diverge at infinity. This is known as the Whitehead paradox in the literature (Whitehead 1889). In the vicinity of the system, indicated as the Stokes or inner regime, the viscous effects are dominant. However, there exists an outer region as $r>O\left(R e^{-1}\right)$, in which the strength of the inertial and viscous forces are comparable regardless of the low Reynolds number approximation. Subsequently, the Stokes solution is ineffectual. Oseen (1910) fixed this paradox by identifying that the perturbation solution has a singularity at the far field and solved this by introducing a different length scale to rescale the governing equations. Proudman \& Pearson (1957) were the first to utilize the approach of singular perturbation and the matched asymptotic expansion to solve the classical problem of low Reynolds number flow around a solid sphere and a circular cylinder. Later, in view of this, the inertial effect of a steady flow past a spherical liquid droplet with uniform interfacial tension was first considered by Taylor \& Acrivos (1964).

The velocity fields of a spherical droplet with a non-uniform interfacial tension up to first order in low Reynolds number flow was attempted by Mason \& Moremedi (2011). Further, several studies have numerically investigated the Marangoni transport of droplets in presence of the convective effects of fluid inertia (Li \& Mao 2001; Bäumler 2014; Blyth \& Pozrikidis 2014; Shardt, Masoud \& Stone 2016; Seric, Afkhami \& Kondic 2018). Nevertheless, much less is known about how the inertial effect influences the swimming characteristics of a Marangoni droplet despite being relevant for artificial systems. A primary feature of self-induced Marangoni propulsion is to relate the prescribed surface 
activity to the migration velocity, which is useful for developing an artificial swimmer, and analyse how it is affected by the many factors of the surrounding medium, including the Reynolds number. Additionally, in the case of active suspensions, it is equally important to understand how the corresponding power dissipation and the swimming efficiency are controlled by the Reynolds number.

Following the classical solutions of Proudman \& Pearson (1957) and Taylor \& Acrivos (1964) of improving the Stokes solution for a flow past a solid and a fluid sphere, respectively, in the present study we carefully utilize the matched asymptotic analysis of a spherical droplet driven by interfacial Marangoni flow at small, but non-zero Reynolds number and predict the effect of the convective inertia on the swimming velocity, power dissipation and swimming efficiency of the droplet swimmer. As mentioned earlier, the variation in the interfacial tension can arise due to different physical mechanisms. In the present work, we prescribe a generic description of the Marangoni effect across the surface of the spherical droplet independently of how the variability in the interfacial tension might have been induced. In this regard, we note that several other studies have examined the self-propulsion of solid bodies at small, but non-zero, Reynolds numbers, and have investigated the relevant swimming properties (Khair \& Chisholm 2011; Wang \& Ardekani 2011; Chisholm et al. 2016). In this work, we probe the answers to similar questions for a Marangoni-driven axisymmetric droplet swimmer. Towards this, we explicitly calculate the interior and exterior streamfunctions and the associated swimming velocity up to the second order in Reynolds number and correlate the results to the swimming characteristics of a solid squirmer (Lighthill 1952; Blake 1971).

The paper is organized as follows. In $\S 2$, we formulate the problem by introducing the governing equations and the related boundary conditions for Marangoni propulsion. In $\S 3$, we employ the singular perturbation and the method of matched asymptotics to determine the analytical solution up to the first and second orders in Reynolds number. In $\S \S 4$ and 5, we discuss the impact of the Reynolds number on the swimming characteristics of the droplet. We provide the main conclusions in $\S 6$.

\section{Mathematical model}

We consider a spherical fluid droplet of radius $a$ and viscosity $\mu^{i}$ which is moving with a velocity $U$ due to an inhomogeneous surface tension $\gamma$ at the interface in an unbounded stationary Newtonian fluid with a viscosity $\mu^{e}$. The fluids are of different viscosities and are assumed to be completely immiscible and incompressible. We assume that the resulting flow in the exterior domain $(r>a)$ due to the presence of the fluid droplet, is a steady low Reynolds number flow with velocity and pressure fields $\left(v^{e}, p^{e}\right)$, while the velocity and pressure fields interior $(r<a)$ to the droplet $\left(\boldsymbol{v}^{i}, p^{i}\right)$ are assumed to be essentially viscous, i.e. at the zero Reynolds number regime. In view of these assumptions, the flow fields exterior as well as interior to the droplet satisfy the Navier-Stokes and Stokes equations, respectively, as (Kim \& Karrila 2005)

$$
\begin{gathered}
\operatorname{Re}\left(\boldsymbol{v}^{e} \cdot \nabla\right) \cdot \boldsymbol{v}^{e}=-\nabla p^{e}+\nabla^{2} \boldsymbol{v}^{e}, \quad \nabla \cdot \boldsymbol{v}^{e}=0, \\
0=-\nabla p^{i}+\nabla^{2} \boldsymbol{v}^{i}, \quad \nabla \cdot \boldsymbol{v}^{i}=0,
\end{gathered}
$$

where $R e=U a \rho^{e} / \mu^{e}<1$ is the Reynolds number associated with the exterior fluid with density $\rho^{e}$. The above velocity and pressure fields are presented in a non-dimensionalized form via $\tilde{\boldsymbol{v}}^{e, i}=\boldsymbol{v}^{e, i} / \boldsymbol{U}, \tilde{\boldsymbol{r}}=r / a, \tilde{\boldsymbol{\nabla}}=a \boldsymbol{\nabla}$ and $\tilde{p}^{e, i}=a p^{e, i} / \mu^{e, i}|\boldsymbol{U}|$. We have further dropped the tilde notation for convenience. We now solve the problem within the reference frame moving with velocity $\boldsymbol{U}$, which is the migration velocity of the droplet. 
Therefore, the exterior velocity field is expected to meet the migration velocity of the droplet in the limit $r \rightarrow \infty$. The inhomogeneous surface tension $(\gamma)$ across the interface is balanced by a stress jump at the fluid-fluid interface. Consequently, the boundary condition corresponding to the tangential stresses are formulated as (Saville 1973; Levan 1981)

$$
\boldsymbol{\Sigma}_{\hat{n} \hat{t}}^{e}-\boldsymbol{\Sigma}_{\hat{n} \hat{t}}^{i}=\nabla_{s} \gamma \cdot \hat{\boldsymbol{t}}, \quad r=a,
$$

where $\boldsymbol{\Sigma}^{e}, \boldsymbol{\Sigma}^{i}$ are the stress tensors corresponding to the external and internal fluids; $\hat{\boldsymbol{n}}$ and $\hat{\boldsymbol{t}}$ are the relevant normal and tangential unit vectors on the surface of the droplet. Further, we expand the inhomogeneous axisymmetric surface tension as $\gamma(\theta)=\sum_{n=1}^{\infty} \gamma_{n} P_{n}(\cos \theta)$ with the strength $\gamma_{n}$ and $P_{n}(\cos \theta)$ are the Legendre polynomials. Now, (2.3) can be simplified in dimensionless form as

$$
\lambda \boldsymbol{\Sigma}_{\hat{n} \hat{t}}^{e}-\Sigma_{\hat{n} \hat{t}}^{i}=T_{s} \cdot \hat{t}, \quad r=1,
$$

where $\lambda=\mu^{e} / \mu^{i}$ is the viscosity ratio of the exterior fluid to the interior. The dimensionless stress field $T_{s}$ developed at the interface of the droplet given by

$$
T_{s}=\sum_{n=1}^{\infty} \frac{2}{(n+1)} \frac{\sigma_{n} \lambda}{U} \nabla_{s} P_{n}(\cos \theta),
$$

where $\sigma_{n}=(n+1) a^{2} \gamma_{n} / 2 \mu^{e}$ denotes the corresponding strength. It is evident from the prescribed definition of $\boldsymbol{T}_{s}$ that we have characterized the surface stress activity in an analogous mathematical structure to the slip velocity in the squirmer model (Lighthill 1952; Blake 1971) for further comparison to this classic model of an axisymmetric spherical micro-swimmer. The normal stress balance can be used to determine the shape change of the droplet, however, we restrict ourselves to the case of a spherical droplet. Although, Taylor \& Acrivos (1964) have shown that the deformation occurs for a spherical fluid droplet at first order in Reynolds number, however, in the case of Marangoni flows, the interfacial tension distribution allows the droplet to remain spherical as it induces an additional surface force across the interface (Stone \& Leal 1990; Hu \& Lips 2003). Furthermore, the surface of the droplet is impenetrable and the tangential fluxes are continuous across the fluid-fluid interface. Therefore, the associated conditions of immiscibility and continuity of the fluids lead to the following conditions for the normal and tangential velocity components, respectively,

$$
\begin{gathered}
\boldsymbol{v}^{e} \cdot \hat{n}=\boldsymbol{v}^{i} \cdot \hat{n}=0, \\
\boldsymbol{v}^{e} \cdot \hat{\boldsymbol{t}}-\boldsymbol{v}^{i} \cdot \hat{\boldsymbol{t}}=0 .
\end{gathered}
$$

\section{Analytical solution of the boundary value problem}

In a simplified approach, we have regarded the migration of the droplet as an axisymmetric motion, and the associated streamfunctions $\psi^{i, e}$ are utilized to describe the flow fields interior and exterior to the droplet, respectively (Happel \& Brenner 1983). Correspondingly, the radial and the tangential components of velocity fields are expressed in terms of the streamfunction as

$$
v_{r}^{i, e}=-\frac{1}{r^{2}} \frac{\partial \psi^{i, e}}{\partial \eta}, \quad v_{\theta}^{i, e}=-\frac{1}{r\left(1-\eta^{2}\right)^{1 / 2}} \frac{\partial \psi^{i, e}}{\partial r},
$$

where $\eta=\cos \theta$. The above streamfunctions are non-dimensionalized as $\psi^{i, e}=\psi^{i, e} / a^{2} U$. Therefore, substituting $(3.1 a, b)$ in the momentum equations, (2.1) and (2.2) can be 
manifested as (Proudman \& Pearson 1957)

$$
\begin{gathered}
\frac{1}{r^{2}} \frac{\partial\left(\psi^{e}, E_{r}^{2} \psi^{e}\right)}{\partial(r, \eta)}+\frac{2}{r^{2}} E_{r}^{2} \psi^{e} L_{r} \psi^{e}=\frac{1}{R e} E_{r}^{4} \psi^{e}, \\
E_{r}^{4} \psi^{i}=0,
\end{gathered}
$$

where $E_{r}^{2}=\partial^{2} / \partial r^{2}+\left(1-\eta^{2}\right) / r^{2} \partial^{2} / \partial \eta^{2} \quad$ and $\quad L_{r}=\eta /\left(1-\eta^{2}\right) \partial / \partial r+1 / r \partial / \partial \eta$. Correspondingly, the far-field $(r \rightarrow \infty)$ and the near-field $(r=1)$ boundary conditions can be expressed in terms of the streamfunction as

$$
\psi^{e} \rightarrow \frac{1}{2} r^{2}\left(1-\eta^{2}\right) \quad \text { as } r \rightarrow \infty,
$$

and at $r=1$,

$$
\begin{gathered}
\psi^{e}=\psi^{i}=0, \\
\frac{\partial \psi^{e}}{\partial r}-\frac{\partial \psi^{i}}{\partial r}=0, \\
\lambda \frac{\partial}{\partial r}\left(\frac{1}{r^{2}} \frac{\partial \psi^{e}}{\partial r}\right)-\frac{\partial}{\partial r}\left(\frac{1}{r^{2}} \frac{\partial \psi^{i}}{\partial r}\right)=-\left(1-\eta^{2}\right)^{1 / 2} \sigma_{1} \lambda(1+2 \sigma \eta) .
\end{gathered}
$$

In the induced stress field $T_{s}$, we have asserted the first two terms $(n=2)$ of the general series (see (2.5)) to proceed with the calculation. Accordingly, we have defined $\sigma=\sigma_{2} / \sigma_{1}$ as the relative strength of the first two modes. The flow around the droplet is analysed for small Reynolds number $R e$; thus, it is required that we must have $\sigma R e<1$ in addition to the condition $R e<1$.

\subsection{Singular perturbation technique}

It may be noted that the available Stokes solution of the Marangoni propulsion of a fluid droplet does not provide a valid correction when the viscous and inertial effects are comparable (Whitehead 1889). The solution breaks down in the limit $r \geq O\left(R e^{-1}\right)$ under the aforementioned boundary conditions. In this context, the problem of low Reynolds number flow around the Marangoni droplet may be considered as a singular perturbation problem (Van Dyke 1975; Hsiao \& MacCamy 1982). Consequently, we adopt the singular perturbation technique followed by the method of matched asymptotic (Kaplun 1957; Proudman \& Pearson 1957) to analytically obtain the asymptotic expansions of the governing streamfunctions of the interior and exterior fluids and analyse the kinematics and energetics of a Marangoni-driven droplet (figure 1).

\subsubsection{Inner or Stokes regime}

In the matched asymptotic expansions, we decompose the continuous phase of the fluid $(r>1)$ into two separate overlapping regions, the Stokes or the inner regime and the Oseen or outer regime. In the Stokes regime, where $r \sim O(1)$ and $R e<1$, we consider that the viscous effects are dominant and the exterior streamfunction is manifested as

$$
\psi^{e}=f_{0}(\operatorname{Re}) \psi_{0}^{e}+f_{1}(\operatorname{Re}) \psi_{1}^{e}+\ldots,
$$

such that $f_{n+1}(R e) / f_{n}(R e) \rightarrow 0$ as $R e \rightarrow 0$. Also, we have assumed that the fluid interior $(r<1)$ to the droplet is highly viscous and obeys the Stokes equation. Consequently, we 


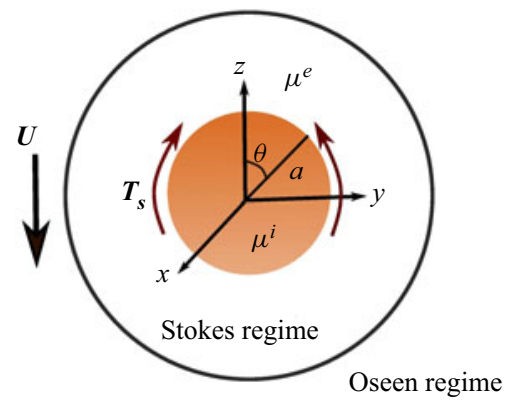

FIGURE 1. The schematic representation of the droplet (of viscosity $\mu^{i}$ ) with an interfacial stress field $T_{s}$ in a low Reynolds number fluid of viscosity $\mu^{e}\left(<\mu^{i}\right)$. A fictitious boundary separates the Stokes and Oseen regimes; $U$ indicates the far-field migration velocity of the droplet in the Oseen regime.

adopt a similar expansion for the interior streamfunction $\psi^{i}$ as

$$
\psi^{i}=f_{0}(R e) \psi_{0}^{i}+f_{1}(R e) \psi_{1}^{i}+\ldots,
$$

where we have used the same expansion coefficients that were used in the exterior streamfunction for simplifying the calculations. The inner expansion of the streamfunctions $\psi^{e}$ and $\psi^{i}$, i.e. (3.8) and (3.9), must satisfy the equations (3.2) and (3.3), respectively, along with the boundary conditions (3.5)-(3.7). The inner expressions are valid in the Stokes regime, therefore, we are restricted to adopt the far-field condition (as in (3.4)) directly. However, the contribution of the far field in the inner expansions of the exterior and interior streamfunctions $\left(\psi^{e}\right.$ or $\left.\psi^{i}\right)$ are later obtained through the matching condition with the outer expansion corresponding to the Oseen region (Proudman \& Pearson 1957).

\subsubsection{Outer or Oseen regime}

In the outer (Oseen) region, where $r \sim O\left(\operatorname{Re}^{-1}\right)$, the inertial term $\operatorname{Re}\left(\boldsymbol{v}^{e} \cdot \nabla\right) \cdot \boldsymbol{v}^{e}$ from the Navier-Stokes equation ((2.1)) is not negligible and, hence, in this region the inner expansion of the exterior streamfunction $((3.8))$ is not valid. This can be avoided by using a suitable length scale to rescale the equation (Oseen 1910). Accordingly, the Oseen variables are defined as

$$
\hat{r}=r \operatorname{Re}, \quad \Psi^{e}=\operatorname{Re}^{2} \psi^{e},
$$

and a straightforward substitution will ascertain (3.2) that

$$
\frac{1}{\hat{r}^{2}} \frac{\partial\left(\Psi^{e}, E_{\hat{r}}^{2} \Psi^{e}\right)}{\partial(\hat{r}, \eta)}+\frac{2}{\hat{r}^{2}} E_{\hat{r}}^{2} \Psi^{e} L_{\hat{r}} \Psi^{e}=E_{\hat{r}}^{4} \Psi^{e},
$$

where the operators $E_{\hat{r}}^{2}$ and $L_{\hat{r}}$ are similar to those defined in the former section. The corresponding far-field condition ((3.4)) takes the form

$$
\Psi^{e} \rightarrow \frac{1}{2} \hat{r}^{2}\left(1-\eta^{2}\right) \quad \text { as } \hat{r} \rightarrow \infty .
$$

As before, the exterior streamfunction in the Oseen region is expressed as

$$
\Psi^{e}=F_{0}(R e) \Psi_{0}^{e}+F_{1}(R e) \Psi_{1}^{e}+\ldots,
$$


where $F_{n+1}(R e) / F_{n}(R e) \rightarrow 0$ as $R e \rightarrow 0$.

\subsection{Leading-order calculation}

The leading-order solution of the Stokes expansion of the interior streamfunction $(r<1)$ satisfies the Stokes equation $E_{r}^{4} \psi_{0}^{i}=0$. Therefore, an appropriate solution which is regular at the origin $(r \rightarrow 0)$ is given by Happel \& Brenner (1983)

$$
\psi_{0}^{i}=\sum_{n=1}^{\infty}\left(C_{n}^{i} r^{n+1}+D_{n}^{i} r^{n+3}\right) Q_{n}(\eta)
$$

where $C_{n}^{i}$ and $D_{n}^{i}$ are the unknown coefficients, and $Q_{n}(\eta)=\int_{-1}^{\eta} P_{n}\left(\eta^{\prime}\right) d \eta^{\prime}$. Likewise, the leading order of the Stokes expansion of the exterior streamfunction (3.8) also satisfies $E_{r}^{4} \psi_{0}^{e}=0$, thus, the solution can be computed as

$$
\psi_{0}^{e}=C_{1}^{e} r^{2} Q_{1}(\eta)+\sum_{n=1}^{\infty}\left(A_{n}^{e} r^{-n}+B_{n}^{e} r^{2-n}\right) Q_{n}(\eta) .
$$

It may be noted that the above solution of the exterior streamfunction is obtained by avoiding the singularities and in view of the uniform far-field streamfunction in the limit $r \rightarrow \infty$. It is evident that, concerning the uniform far field and the prescribed surface activity $((3.7))$, the higher-order $(n \geq 3)$ terms in the general expansions of the interior and exterior streamfunctions are zero. Correspondingly, the unknown coefficients $\left(C_{n}^{i}, D_{n}^{i}\right)$ and $\left(A_{n}^{e}, B_{n}^{e}\right)$ can be determined by employing the aforesaid boundary conditions at the fluid-fluid interface. However, the coefficient of far field, i.e. $C_{1}^{e}$, can be determined by matching the leading-order solutions of the Stokes and Oseen regimes. Thus, the general solutions of the leading-order interior $(r<1)$ and exterior $(r>1)$ streamfunctions can be obtained as

$$
\begin{aligned}
\psi_{0}^{i}= & \lambda \frac{\left(3 C_{1}^{e}+2 \sigma_{1}\right)}{6(1+\lambda)}\left(r^{4}-r^{2}\right) Q_{1}(\eta)-\frac{2 \sigma_{2} \lambda}{(5 U(1+\lambda))}\left(r^{2}-r^{4}\right) Q_{2}(\eta), \\
\psi_{0}^{e}= & \left(C_{1}^{e} r^{2}-\frac{\left((3+2 \lambda) C_{1}^{e}-2 \sigma_{1} \lambda / 3 U\right)}{2(1+\lambda)} r+\frac{\left(C_{1}^{e}-2 \sigma_{1} \lambda / 3 U\right)}{2(1+\lambda) r}\right) Q_{1}(\eta) \\
& +\frac{2 \sigma_{2} \lambda}{(5 U(1+\lambda))}\left(1-\frac{1}{r^{2}}\right) Q_{2}(\eta) .
\end{aligned}
$$

Now, the leading-order solution in the Oseen regime should necessarily agree with the uniform far-field condition at infinity. Consequently, we indicate $\Psi_{0}^{e}$ as (from (3.12))

$$
\Psi_{0}^{e}=-\hat{r}^{2} Q_{1}(\eta) \quad \text { with } F_{0}(R e)=1
$$

\subsubsection{Determination of $C_{1}^{e}$}

In order to match the leading-order solutions of the Stokes and Oseen regimes, we further express (3.17) in terms of the Oseen variables and consider the limit $R e \rightarrow 0$. This would imply that $C_{1}^{e}=-1$. Thus, Stokes solution for the Marangoni-driven droplet is recovered (Balasubramaniam \& Subramanian 2000; Schmitt \& Stark 2016b). 


\subsection{First-order corrections}

Under the assumption of negligible inertial effect, the first order of the interior streamfunction gives rise to the Stokes equation, i.e. $E_{r}^{4} \psi_{1}^{i}=0$, which admits a general solution as been mentioned in (3.14). On the other hand, after substituting (3.8) and (3.13) in (3.2) and (3.11), respectively, the governing equations for the first-order exterior streamfunction in the Stokes and Oseen regimes, respectively, can be obtained as

$$
\begin{gathered}
\frac{f_{1}(R e)}{R e} E_{r}^{4} \psi_{1}^{e}=\frac{1}{r^{2}} \frac{\partial\left(\psi_{0}^{e}, E_{r}^{2} \psi_{0}^{e}\right)}{\partial(r, \eta)}+\frac{2}{r^{2}} E_{r}^{2} \psi_{0}^{e} L_{r} \psi_{0}^{e}, \\
E_{\hat{r}}^{4} \Psi_{1}^{e}=\frac{\left(1-\eta^{2}\right)}{\hat{r}} \frac{\partial\left(E_{\hat{r}}^{2} \Psi_{1}^{e}\right)}{\partial \eta}+\eta \frac{\partial\left(E_{\hat{r}}^{2} \Psi_{1}^{e}\right)}{\partial \hat{r}} .
\end{gathered}
$$

In the following, we set $f_{1}(R e)=R e$ without the loss of generality (Proudman \& Pearson 1957). Further, by substituting (3.17) in (3.19), the governing equation of $\psi_{1}^{e}$ can be simplified to,

$$
\begin{aligned}
E_{r}^{4} \psi_{1}^{e}= & \left(\frac{6 A_{2}^{e} B_{1}^{e}}{5 r^{4}}+\frac{6 A_{2}^{e}}{5 r^{6}}\left(B_{1}^{e}+6 A_{1}^{e}\right)\right) Q_{1}(\eta) \\
& +\left(\frac{6 B_{1}^{e}}{r^{2}}-\frac{6\left(B_{1}^{e}\right)^{2}}{r^{3}}-\frac{6 A_{1}^{e} B_{1}^{e}}{r^{5}}-\frac{24\left(A_{2}^{e}\right)^{2}}{7 r^{5}}+\frac{48\left(A_{2}^{e}\right)^{2}}{7 r^{4}}\right) Q_{2}(\eta) \\
& +\left(\frac{144 A_{2}^{e} B_{1}^{e}}{5 r^{4}}-\frac{24 A_{2}^{e}}{r^{3}}+\frac{84 A_{2}^{e} A_{1}^{e}}{5 r^{6}}-\frac{36 A_{2}^{e} B_{1}^{e}}{5 r^{6}}\right) Q_{3}(\eta) \\
& +\left(\frac{120\left(A_{2}^{e}\right)^{2}}{7 r^{7}}-\frac{144\left(A_{2}^{e}\right)^{2}}{7 r^{7}}\right) Q_{4}(\eta)
\end{aligned}
$$

where $A_{1}^{e}, B_{1}^{e}, A_{2}^{e}$ are the coefficients of the leading-order solution of the exterior streamfunction in the Stokes regime (see (3.15)) which have been determined using the boundary conditions mentioned in $\S 3.2$. It may be noted that without explicit substitution of the coefficients, the structure of inhomogeneous part of (3.21) appears identical to the analysis by Wang \& Ardekani (2011) for the inertial effects on a squirmer. Accordingly, we write the general solution of (3.21) as $\psi_{1}^{e}=\sum_{n=1}^{4} g_{n}(r) Q_{n}(\eta)$, where

$$
\begin{gathered}
g_{1}(r)=\left(\frac{\alpha_{1}^{e}}{r}+\beta_{1}^{e} r+\gamma_{1}^{e} r^{2}-\frac{3}{20} A_{2}^{e} B_{1}^{e}+\frac{A_{2}^{e}}{10 r^{2}}\left(A_{1}^{e}+B_{1}^{e} / 6\right)\right), \\
g_{2}(r)=\left(\frac{\alpha_{2}^{e}}{r^{2}}+\beta_{2}^{e}-\frac{\left(B_{1}^{e}\right)^{2}}{4} r+\frac{B_{1}^{e}}{4} r^{2}+\left(\frac{A_{1}^{e} B_{1}^{e}}{4}+\frac{\left(A_{2}^{e}\right)^{2}}{7}\right) \frac{1}{r}+\frac{\left(A_{2}^{e}\right)^{2}}{21 r^{3}}\right), \\
g_{3}(r)=\left(\frac{\alpha_{3}^{e}}{r^{3}}+\frac{\beta_{3}^{e}}{r^{1}}+\frac{2}{5} A_{2}^{e} B_{1}^{e}-\frac{A_{2}^{e}}{5} r+\frac{1}{20 r^{2}}\left(-7 A_{2}^{e} A_{1}^{e}+3 A_{2}^{e} B_{1}^{e}\right)\right), \\
g_{4}(r)=\left(\frac{\alpha_{4}^{e}}{r^{4}}+\frac{\beta_{4}^{e}}{r^{2}}-\frac{\left(A_{2}^{e}\right)^{2}}{7 r}-\frac{3\left(A_{2}^{e}\right)^{2}}{14 r^{3}}\right) .
\end{gathered}
$$

The above expressions are determined considering the fact that the streamfunction in the Stokes regime should not contain higher-order terms (in $r$ ) than the uniform far-field streamfunction. All the unknown coefficients $\left(\alpha_{n}^{e}, \beta_{n}^{e}\right)$ and $\left(C_{n}^{i}, D_{n}^{i}\right)$ at $O(R e)$ are to be determined from the boundary conditions mentioned in (3.5)-(3.7). Further, 
to determine $\gamma_{1}^{e}$, we match the coefficient of the highest order of $r$ in $\psi_{1}^{e}$ (i.e. $r^{2}$ ) to the same in the first-order solution in the Oseen regime, which we determine in what follows.

According to Goldstein (1929), (3.20) can be solved using a transformation $E_{\hat{r}}^{2} \Psi_{1}^{e}=$ $\mathrm{e}^{\hat{r} \eta / 2} \phi$. Hence, the general solution of $\Psi_{1}^{e}$ that vanishes at infinity and $\eta= \pm 1$ can be determined as (Proudman \& Pearson 1957) (for details see appendix A)

$$
\Psi_{1}^{e}=-B_{1}^{e}(1+\eta)\left(1-\exp \left(-\frac{\hat{r}(1-\eta)}{2}\right)\right) .
$$

\subsubsection{Determination of $\gamma_{1}^{e}$}

From the first-order solutions in the Stokes regime (i.e. $\psi_{1}^{e}$ ) and Oseen regime (i.e. $\Psi_{1}^{e}$ ), the coefficient of the highest-order term $r^{2}$ or $\hat{r}^{2}$ can be identified as

$$
\begin{aligned}
& \Psi_{1}^{e} \approx \frac{B_{1}^{e}}{8}\left(1-\eta^{2}\right)(1+\eta) \hat{r}^{2}, \\
& \psi_{1}^{e} \approx\left(\gamma_{1}^{e} r^{2} Q_{1}+\frac{B_{1}^{e}}{4} r^{2} Q_{2}\right) .
\end{aligned}
$$

In view of the matching condition, (3.25) can be expressed in term of the Oseen variables. Correspondingly, (3.24) should agree with (3.25), and $\gamma_{1}^{e}$ which determines $\gamma_{1}^{e}=-B_{1}^{e} / 4$. This is supported by the results of Wang \& Ardekani (2011).

\subsection{Second-order correction}

From (3.2) and (3.11), the governing equations for the second-order exterior streamfunction in the inner and Oseen regimes are respectively given by

$$
\begin{gathered}
E^{4} \psi_{2}^{e}=\frac{1}{r^{2}}\left(\frac{\partial\left(\psi_{0}^{e}, E^{2} \psi_{1}^{e}\right)}{\partial(r, \eta)}+\frac{\partial\left(\psi_{1}^{e}, E^{2} \psi_{0}^{e}\right)}{\partial(r, \eta)}\right)+\frac{2}{r^{2}}\left(E^{2} \psi_{0}^{e} L \psi_{1}^{e}+E^{2} \psi_{1}^{e} L \psi_{0}^{e}\right), \\
E_{\hat{r}}^{4} \Psi_{2}^{e}=\frac{\left(1-\eta^{2}\right)}{\hat{r}} \frac{\partial\left(E_{\hat{r}}^{2} \Psi_{2}^{e}\right)}{\partial \eta}+\eta \frac{\partial\left(E_{\hat{r}}^{2} \Psi_{2}^{e}\right)}{\partial \hat{r}},
\end{gathered}
$$

where we have set $f_{2}(R e)=R e^{2}$ in the inner regime without loss of generality. Further, using the solutions for $\psi_{0}^{e}$ and $\psi_{1}^{e}((3.17)$ and (3.22)), the inhomogeneous part (right-hand side) of (3.26) can be obtained in accordance with the angular dependency $Q_{n}(\eta)$. It may be noted that, due to the arduous structure of the inhomogeneous part, we pursue the solution of the second-order streamfunction $\psi_{2}^{e}$ up to the first mode $(n=1)$, i.e. the contribution of the streamfunction that is necessary for determining the migration velocity. A straightforward calculation of the complementary solution and the particular integral yields

$$
\begin{aligned}
\psi_{2}^{e}= & \left(\frac{a_{1}^{e}}{r}+b_{1}^{e} r+c_{1}^{e} r^{2}-\frac{3}{20}\left(A_{2}^{e} \beta_{1}^{e}-\frac{A_{1}^{e} B_{1}^{e}}{2}-B_{1}^{e} \beta_{2}^{e}\right)\right. \\
& \left.+\left(-\frac{\alpha_{1}^{e}}{50}-\frac{\beta_{1}^{e}}{300}-\frac{A_{1}^{e} \beta_{2}^{e}}{10}+\frac{B_{1}^{e} \alpha_{2}^{e}}{60}\right) \frac{1}{r^{2}}-\frac{\left(A_{1}^{e}\right)^{2} B_{1}^{e}}{200 r^{3}}\right) Q_{1}(\eta),
\end{aligned}
$$


where the unknown coefficients $a_{1}^{e}$ and $b_{1}^{e}$ can be determined from the boundary conditions prescribed in (3.5)-(3.7) and the coefficient $c_{1}^{e}$ from the matching condition with the second-order streamfunction in the Oseen regime.

Regarding this, we notice that the governing equation for $\Psi_{2}^{e}$ in the Oseen regime is identical to (3.20) with $\Psi_{1}^{e}$ replaced by $\Psi_{2}^{e}$. Therefore, a solution up to the first mode $\left(Q_{1}(\eta)\right)$ is analogous to (A 1). Additionally, the first mode contribution of the derived inner streamfunction (i.e. $\psi^{e} \sim \psi_{0}^{e}+\operatorname{Re} \psi_{1}^{e}$ ) in the Oseen regime is quantified as $E^{2} \psi^{e} \sim$ $-2 \beta_{1}^{e} Q_{1}(\eta) / r$ at $O\left(R e^{2}\right)$. Consequently, the unknown coefficient $\mathcal{A}_{1}$ for the second-order streamfunction in the Oseen regime is retrieved as $\mathcal{A}_{1}=-\beta_{1}^{e}$. Furthermore, an appropriate matching condition yields $c_{1}^{e}=-\beta_{1}^{e} / 4$.

In this context, the fundamental difference between a passive and an active spherical body was noted by Khair \& Chisholm (2011). For any creeping flow past a passive droplet, due to the existence of a uniform far-field velocity of the ambient medium, a finite net force acts on the surface of the body, whereas, for an active self-propelling system, due to the force-free nature of the motion, the far-field velocity typically indicates the migration velocity of the swimmer. Apparently, by virtue of this, the logarithm terms $\left(O\left(R e^{2} \ln R e\right)\right)$, which appear in the second order in the Stokes expansion for a passive system, do not appear at $O\left(R e^{2}\right)$ (Khair \& Chisholm 2011) for active systems. This summarizes the complete calculation of the streamfunctions in the Stokes and Oseen regimes for a Marangoni-driven droplet up to $O(R e)$ and an approximated first mode solution for the exterior streamfunction at $O\left(R e^{2}\right)$. In the following sections, we allude the significance of this inertial forcing encountered by the droplet on its swimming motility, flow fields and energy expenditures utilizing the complete general solutions up to $O(R e)$ and comment on the distinctness of the first-order and second-order corrected migration velocities.

\section{Results: first-order correction}

\subsection{Drag on the surface of the droplet}

For evaluation of the drag on the surface of the droplet, we proceed with the streamfunction $\left(\psi^{e}=\psi_{0}^{e}+\operatorname{Re} \psi_{1}^{e}\right)$ in the Stokes or inner regime which is well founded in the vicinity of the droplet. For axisymmetric swimming, the non-zero component of the drag force acting in the $z$-direction is given by

$$
D=\int_{s}\left(\Sigma_{r r} \cos \theta-\Sigma_{r \theta} \sin \theta\right) \mathrm{d} s,
$$

where $\mathrm{d} s$ is the surface element of the droplet. Having computed the velocity fields from the streamfunction, we further obtain the corresponding stresses and arrive at the following expression for dimensionless drag force

$$
D \approx D_{0}+\operatorname{Re} D_{1},
$$

where $D_{0}$ and $D_{1}$ are evaluated as

$$
\begin{gathered}
D_{0}=\frac{2 \pi(3+2 \lambda)}{1+\lambda}\left(1-\frac{1}{U}\right), \\
D_{1}=\frac{\pi(3+2 \lambda)^{2}}{4(1+\lambda)^{2}}\left(1-\frac{1}{U}\left(\frac{11+10 \lambda}{3+2 \lambda}+\frac{\sigma\left(36+67 \lambda+26 \lambda^{2}\right)}{250(1+\lambda)(3+2 \lambda)}\right)-\frac{\sigma(2+\lambda)}{50 U^{2}(1+\lambda)}\right),
\end{gathered}
$$


with $U \rightarrow U / U_{0}$ as the dimensionless velocity of the droplet. We have used $U_{0}=$ $-2 \sigma_{1} \lambda /(3(3+2 \lambda))$ as the reference scale, which is the standard locomotion speed for a Marangoni stress-driven droplet in the limit $R e \rightarrow 0$ (Schmitt \& Stark 2016b). For self-propulsion of the droplet, the net drag $(D)$ must vanish on the surface of the droplet. Consequently, we arrive at the following quadratic equation in $U$,

$$
\begin{aligned}
D= & \left(2+\operatorname{Re} \frac{3+2 \lambda}{4(1+\lambda)}\right) U^{2}-\left(2+\operatorname{Re} \frac{11+10 \lambda}{4(1+\lambda)}+\operatorname{Re} \sigma \frac{\left(36+67 \lambda+26 \lambda^{2}\right)}{250(1+\lambda)^{2}}\right) U \\
& -\operatorname{Re} \sigma \frac{(2+\lambda)(3+2 \lambda)}{50(1+\lambda)^{2}}=0 .
\end{aligned}
$$

The migration velocity $U$ can be determined by solving the above quadratic equation up to $O(R e)$ limit. The approximated solutions can be written as

$$
\begin{gathered}
U_{1} \approx 1+\operatorname{Re}\left(1+\sigma\left(0.134-0.064 \lambda+0.066 \lambda^{2}\right)\right), \\
U_{1}^{*} \approx-\operatorname{Re} \sigma\left(0.06-0.05 \lambda+0.06 \lambda^{2}\right) .
\end{gathered}
$$

It may be noted that the above solutions are obtained subject to the constraint $\lambda<1$ up to $O\left(\lambda^{2}\right)$. This constraint indicates that the interior fluid is more viscous than the exterior, consistent with our prior assumption. Correspondingly, the $\sigma \operatorname{Re} \lambda<1$ and $\sigma \operatorname{Re} \lambda^{2}<1$ conditions are maintained in the further calculations. We observe that $U_{1}^{*}$ (as in (4.7)) vanishes in the limit $R e \rightarrow 0$. Hence, we treat $U_{1}$ (as in (4.6)) as the corrected migration velocity $U$ at $O(R e)$ for the sake of concurrence of the velocity field and the associated swimming characteristics in the limit $R e \rightarrow 0$.

\subsection{Migration velocity}

In the absence of inertial effect, i.e. in $R e \rightarrow 0$ limit, the locomotion speed of the droplet is essentially controlled by the first mode of the surface stress activity. However, (4.6) implies that the second mode of the induced stress field plays a predominant role in the migration velocity of the droplet for finite Reynolds numbers. In figure 2(a), the variation of $U$ with the Reynolds number $R e$ has been depicted for a set of $\sigma$ values. Typically, the inertial effect of the medium reduces the viscous resistance for the droplet and hence the migration velocity is enhanced. In pursuance of comparison with the low Reynolds number characteristics of the squirmers, which is the classical model for analysing the migration of micro-swimmers, a straightforward analysis of the surface flows and the associated stresslet strength of any Marangoni stress-driven flow will imply that $\beta=\beta_{2} / \beta_{1} \sim-\sigma_{2} / \sigma_{1}$ (Schmitt \& Stark 2016b). For squirmers using purely tangential velocity actuation, one can categorize the swimmer as neutral when $\beta=0$, a pusher (or puller) when $\beta$ is negative (or positive). This can be verified by the swimming patterns observed in figure 3 where the streamlines for $\sigma=-5$ are analogous to the swimming pattern of a puller $(\beta>0)$ and vice versa.

For solid squirmers, pushers $(\beta<0)$ have their locomotion speed increased while for pullers $(\beta>0)$, the speed decreases with $R e$. Further, for a neutral $(\beta=0)$ squirmer stresslets are absent in the external medium; hence, the migration velocity remains unaltered by $R e$ (Wang \& Ardekani 2011). In contrast, the observation that the migration velocity of a swimming droplet exhibits an increasing trend with $R e$ for any value of $\sigma$ is intriguing. Similar to a neutral squirmer, for $\sigma=0$, due to the absence of vortices in the exterior medium, the interfacial stress in the direction of migration 

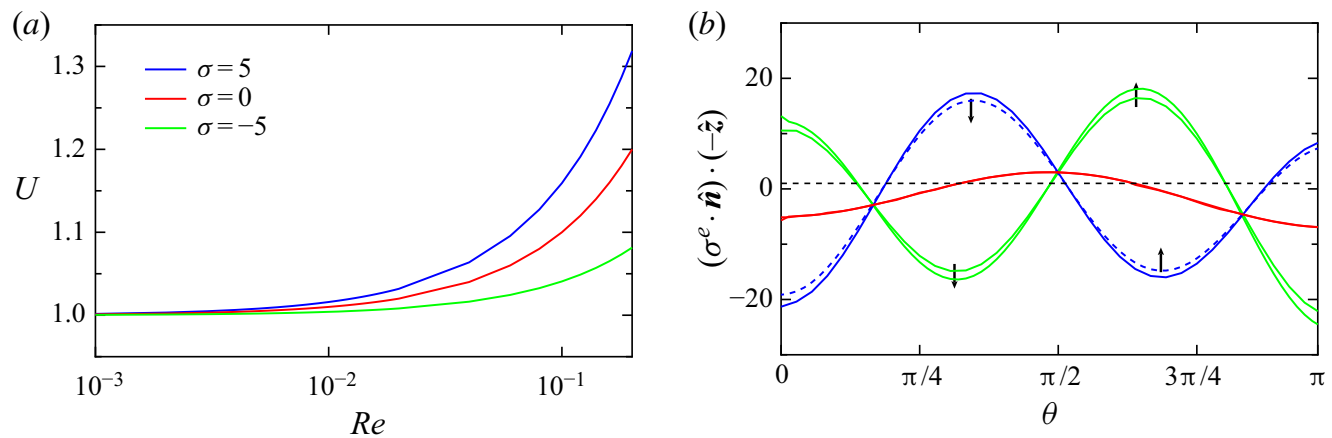

FIgURE 2. (a) The dimensionless migration velocity $(U)$ as a function of $R e$, for different $\sigma$ values fixing $\sigma_{1}=1$ and $\lambda=0.5$. We have varied $R e$ in such a way that the conditions $\sigma R e<1$, $\sigma \operatorname{Re} \lambda<1$ and $\sigma \operatorname{Re} \lambda^{2}<1$ are satisfied. (b) The variation of the surface stress of the spherical droplet swimmer, in the direction of migration, as a function of polar angle $\theta$ for different $\sigma$ values. The solid and dashed lines (green \& blue) represent the stress variation for $\operatorname{Re}=0$ and $R e=0.19$, respectively. For $\sigma=0$, the solid and dashed lines coincide.
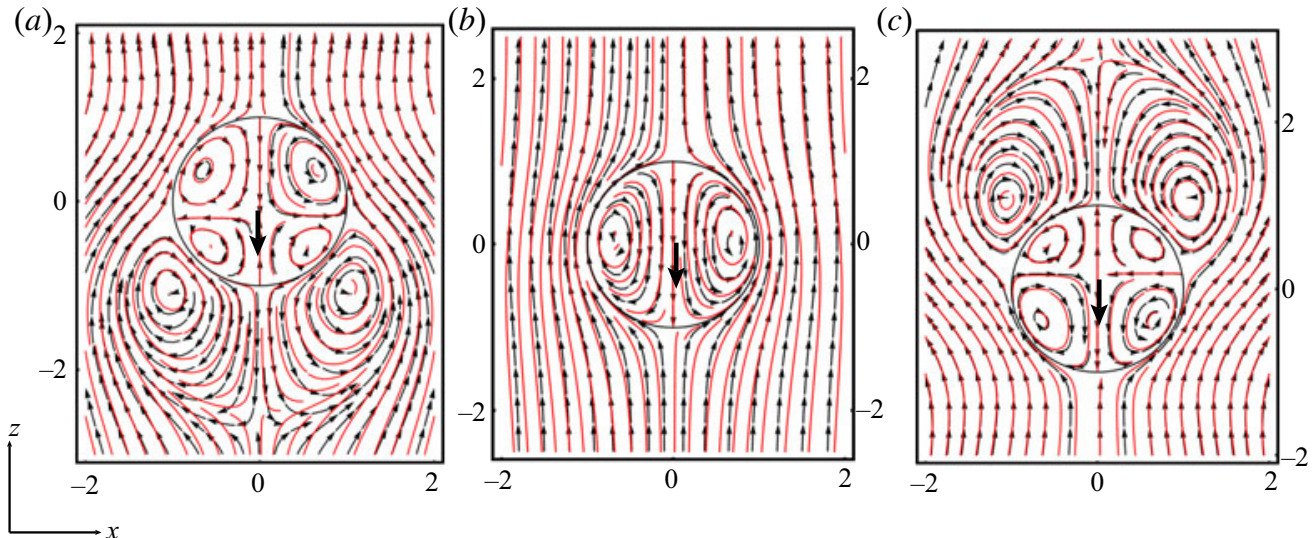

FIGURE 3. The comparison of the streamlines of the velocity field of the droplet swimmer with (a) $\sigma=5$, (b) $\sigma=0$ and (c) $\sigma=-5$. The red lines and the black arrow lines represents the streamlines for $R e=0.19$ and the $R e=0$ limit, respectively at $\lambda=0.5$. The solid black arrow indicates the direction of the migration.

(i.e. negative $z$-direction) remains unaltered at finite $R e$ (see figure $2 b$ ). However, for droplets with $\sigma=0$, vortices are formed interior to the droplet, which gets redistributed due to the inertial effects (see figure $3 b$ ). Therefore, the migration velocity evolves with $R e$. Straightaway, from (4.6) we note that the subsequent increase from the neutral case is relatively higher or lower for $\sigma>0$ or $\sigma<0$, respectively. Figure 3(a) depicts that, for $\sigma=5$, the stresslets or the closed-streamline re-circulatory regions of the external fluid, developed in the direction of the migration, get abbreviated due to finite inertial effects. Subsequently, the interfacial stress in the direction of migration gets reduced for $\sigma>0$ (see figure $2 b$ ) and the migration velocity increases significantly, identical to the behaviour of an equivalent squirmer (with $\beta<0$ ). Surprisingly for $\sigma=-5$, although the external stresslets enlarge due to inertial effect, which successively enhances the interfacial stress (see figure $2 b$ ), the migration velocity increases with $R e$. This is due to the existence of 
(a)

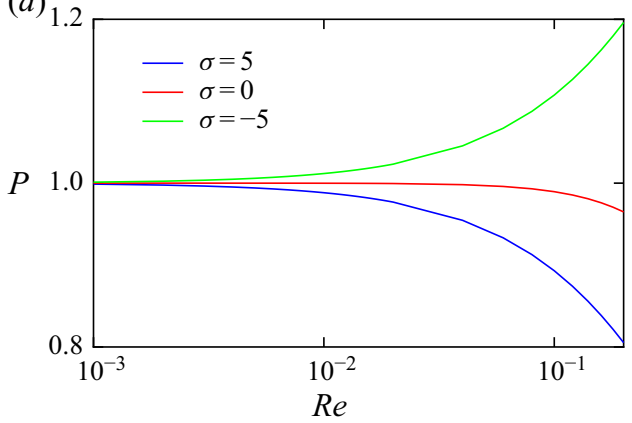

(b)

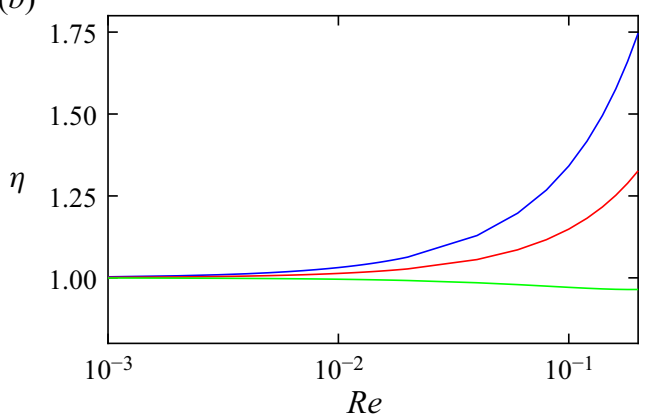

FIGURE 4. (a) The power dissipation and (b) the corresponding efficiency of the droplet swimmer as a function of the Reynolds number $R e$ for different $\sigma$ values fixing $\sigma_{1}=1$ and $\lambda=0.5$. Here, the absolute power and efficiency of the droplet are scaled with the respective values at $R e \rightarrow 0$ limit, i.e. $P \rightarrow P / P_{R e \rightarrow 0}$ and $\eta \rightarrow \eta / \eta_{R e \rightarrow 0}$.

prominent counter rotating vortices of the interior flow in the direction of migration of the droplet (see figure $3 c$ ), which re-acclimate and retaliate the enhanced stress at finite $\operatorname{Re}$ (Mason \& Moremedi 2011). In this regard, we note that the previous counteraction for $\sigma<0$ is inconceivable for an equivalent solid squirmer (with $\beta>0$ ). This alludes to the fact that the droplet swimmers are more malleable at finite $R e$ than its rigid counterparts.

Furthermore, as a consequence of truncation up to $\lambda^{2}$, we observe that Re quadratically couples with $\lambda$ and $\sigma$ (see (4.6)). Correspondingly, the magnitude of net migration velocity $\left(-2 \sigma_{1} \lambda /(3(3+2 \lambda)) U\right.$ increases monotonically as a function of $\lambda$ (within the limit $\lambda<1$ ) with escalation governed by the choice of $\sigma$ at $O(R e)$. This is identical to the qualitative behaviour of $U$ vs. $\lambda$ in the limit $R e \rightarrow 0$. Overall, the above results highlight the fact that within the insisted range $\lambda<1$ the inertial effect of a droplet is invariably lower than the external fluid, therefore both $R e$ and $\lambda$ synergistically enhance the locomotion speed of the droplet swimmer, and a keen choice of $\sigma$ determines the percentage increase.

\subsection{Power dissipation and efficiency}

Primarily, the rate of work done by the swimmer can be quantified by $P=-\int\left(\Sigma^{e} \cdot \hat{\boldsymbol{n}}\right)$. $\boldsymbol{v}^{e} \mathrm{~d} s$, which can be calculated with the help of constitutive relations and the corresponding flow fields of the swimmer. Additionally, using the reciprocal theorem, the viscous dissipation of a spherical body with purely tangential surface activity can be written in terms of the vorticity and the surface velocity of the swimmer (Stone \& Samuel 1996; Masoud \& Stone 2019). Following this, one may infer that with increasing $R e$, the droplet that minimizes the vorticity will also minimize the power dissipation. Figure 4(a) illustrates this trend. The vortices in the vicinity of the droplet when $\sigma<0$ get outweighed for non-zero $R e$, which enhance the corresponding dissipated power with increasing $R e$. Nevertheless, for $\sigma \geq 0$, the dissipated power decreases monotonically due to abbreviation or redistribution of the external or internal vortices, respectively. One might conjecture that this behaviour contradicts the Helmholtz minimum dissipation theorem (Batchelor 1967), which guarantees that a Stokes flow field dissipates less energy than any other incompressible flow field with the same boundary velocities. However, the theorem does not apply here as the far-field boundary velocity is denoted by the migration velocity of the droplet which depends on Reynolds number. 
While the energetic cost of moving through a medium is an essential property of locomotion, for self-propelling systems, it is relevant to investigate how the properties of the swimmer and the surrounding medium influence the efficiency of the locomotion. According to Lighthill (1952), the efficiency $(\eta)$ of a swimmer is defined as the ratio of the power necessary to pull the swimmer at the swimming speed (i.e. $F_{H} U$ ) to the rate of work done $(P)$ by the swimmer, i.e. $\eta=F_{H} U / P$. Here, we consider $F_{H}$ as the hydrodynamic force acting on the spherical droplet at $O(R e)$ limit, which is given by (Taylor \& Acrivos 1964)

$$
F_{H} \sim 2 \pi\left(\frac{3+2 \lambda}{1+\lambda}+R e \frac{(3+2 \lambda)^{2}}{8(1+\lambda)^{2}}\right) .
$$

Note that $F_{H}$ also represents the Hadamard and Rybczynski drag force of a droplet in the limit $R e \rightarrow 0$ (Clift, Grace \& Weber 1978; Sadhal, Ayyaswamy \& Chung 1997). The above expression agrees with the well-established low Reynolds number drag of a solid sphere in the limit $\lambda \rightarrow 0$ (Proudman \& Pearson 1957). Note that the swimming efficiency is systematically higher over the entire range of $\operatorname{Re}$ (see figure $4 b$ ) for $\sigma>0$ and $\sigma=0$. It would be reasonable to expect that droplets with $\sigma>0$ are more efficient than the droplets with $\sigma=0$. Notably, for $\sigma=5, \eta$ is significantly pronounced (more than 1.5 times) in the limit $\sigma R e<1$, whereas for $\sigma=-5$ the efficiency meagrely reduces due to higher power consumption at $O(R e)$. Although for $\sigma<0, U$ increases as a function of $R e$, the output power $F_{H} U$ under performs the dissipated power $P$, which further decreases the efficiency. This reinforces the fact that for a broader class of droplets $(\sigma \geq 0)$ swimming against fully resistive viscous medium is challenging and less efficient (Purcell 1977). When inertia is present, the droplets $(\sigma \geq 0)$ effectively exploit the depleted viscous resistance from the external medium to gain higher motility as well as energetically efficient swimming. This finding is analogous to the efficiency of a solid squirmer in a low Reynolds number flow.

Moreover, our results reveal some noticeable observations on the behaviour of $P$ and $\eta$ with respect to the viscosity ratio $\lambda$ at finite $R e$. Recall that for a droplet with $\sigma=0$, no additional $\lambda$ dependency is witnessed in the corrected migration velocity $\tilde{U}$ (see (4.6)). Consequently, all the associated swimming properties necessarily follow the corresponding Stokesian behaviour (i.e. at $\operatorname{Re} \rightarrow 0$ ) with $\lambda$. Interestingly, for $\sigma>0$, the Stokesian behaviour also persists with increasing $\lambda$ at $O(R e)$ despite an acquired $\lambda^{2}$ reliance. Subsequently, we observe $P$ (or $\eta$ ) monotonically decreases (or increases) with $\lambda$ in the limit $\lambda<1$ (see figure 5). However, for $\sigma<0$, as mentioned earlier that the stresslets generated by the swimmer intensify due to the inertial effect. Consequently, the apparent extent of the swimmer (i.e. swimmer with the associated vortices) increases at finite $R e$. Therefore, towards higher $\lambda$ (within the limit $\lambda<1$ ) the Reynolds number associated with the internal fluid becomes comparable to the Reynolds number of the external fluid. Consequently, the swimmer with $\sigma<0$ dissipates lower power $(P)$ and exhibits higher efficiency $(\eta)$ towards higher $\lambda$ and the foreseen increasing (or decreasing) trend for $\eta$ (or $P$ ) discontinues at higher $\lambda$ (see figure 5).

\subsection{Linear stability analysis of the migration}

In the preceding section, we have shown the impact of the inertial effect on the self-propulsion of the droplet with inhomogeneous surface tension. Towards this, we have asserted the dimensionless velocity $U=U_{1}$ as the coherent solution at $O(R e)$. However, the present system incorporates the nonlinear convective inertial effects of the surrounding medium; this persuades the investigation of the linear stability of drag 
(a)

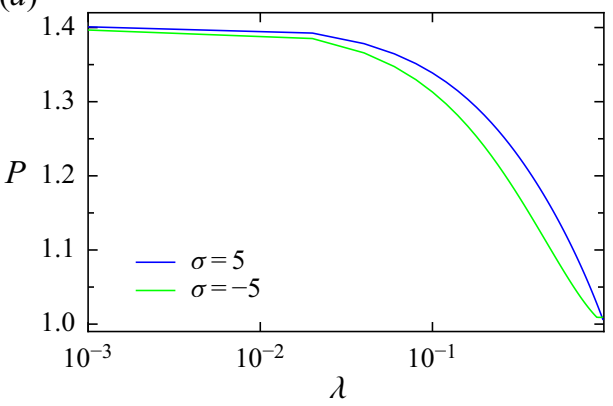

(b)

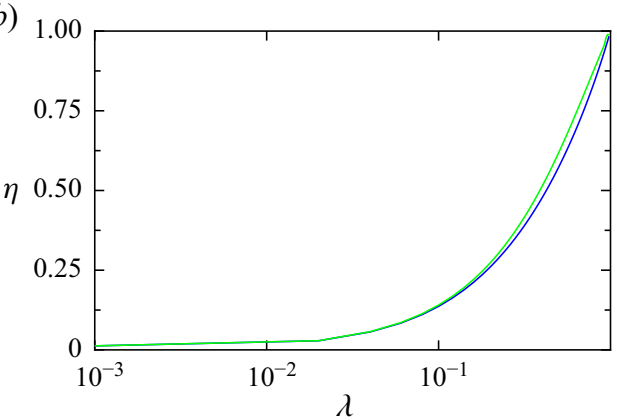

FIGURE 5. (a) The power dissipation and $(b)$ the corresponding efficiency of the droplet swimmer as a function of the viscosity ratio $\lambda$ for different $\sigma$ values at $R e=0.19$. Here, the absolute power and efficiency of the droplet are scaled as $P \rightarrow P / P_{\lambda \rightarrow 1}$ and $\eta \rightarrow \eta / \eta_{\lambda \rightarrow 1}$, respectively.

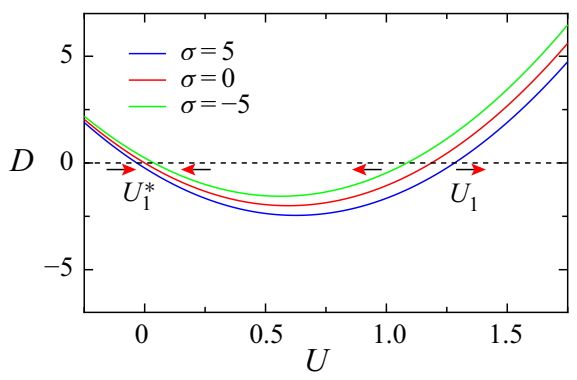

FIGURE 6. The nonlinear drag function $D((4.5))$ as a function of the dimensionless migration velocity $U$ at $R e=0.19, \lambda=0.5$, and at different $\sigma$ values. $U_{1}$ and $U_{1}^{*}$ are the fixed points of the function. The arrows indicate the trend of the function near the fixed points, which also demonstrates the stability.

function $D$ derived in (4.5). Figure 6 illustrates the fixed points of the drag function $D$, which mark the migration velocities $U_{1}$ and $U_{1}^{*}$. We perform the linear stability analysis around the fixed points. We analyse the first derivative of the drag function $D$ at $U_{1}$ and $U_{1}^{*}$, which measures the rate of decay to the corresponding fixed points. Further investigation indicates that at $U=U_{1}, \partial D / \partial U>0$, whereas at $U=U_{1}^{*}$, we have $\partial D / \partial U<0$ for all $\sigma$ values. Interestingly, the negative slope at the latter fixed point establishes $U=U_{1}^{*}$ as an asymptotically stable swimming state of the droplet swimmer, which corresponds to zero migration in $R e \rightarrow 0$ limit. This can be understood as follows.

The external sources for inhomogeneous surface tension i.e. external thermal or chemical gradients, or inhomogeneous coverage of surfactant molecules reduce the local interfacial tension $(\gamma)$, developing the Marangoni flow towards the higher $\gamma$ value. However, the Marangoni flow across the interface induces a short-lived motion of the droplet. In the absence of external forces, neglecting the inertial effect of the medium, the long-time limit leads to an equilibrium state of the droplet. This corresponds to no migration as the droplet achieves a state of uniform interfacial tension (Izri et al. 2014). Nevertheless, for low but finite Reynolds number due to nonlinear convective effect of the surroundings, we proclaim that at a later stage the velocity of the droplet decreases from the initial velocity $U_{1}$ and develops a distinct migration velocity $U_{1}^{*}$ before impending the halt. This asymptotically stable migration velocity is an outcome of the nonlinear 


\begin{tabular}{lcccc} 
& \multicolumn{4}{c}{$(U-1)$} \\
\cline { 2 - 5 }$R e$ & $O(R e)$ & $O\left(R e^{2}\right)$ & $O(R e)$ & $O\left(R e^{2}\right)$ \\
$10^{-4}$ & 0.00016 & 0.00016 & 0.00004 & 0.00004 \\
$10^{-3}$ & 0.00159 & 0.00161 & 0.00041 & 0.00042 \\
$10^{-2}$ & 0.01593 & 0.01732 & 0.00408 & 0.00498 \\
$10^{-1}$ & 0.15925 & 0.29887 & 0.04075 & 0.13134
\end{tabular}

TABLE 1. A direct comparison of the quantitative increase of $U$ at $O(R e)$ and $O\left(R e^{2}\right)$.

interaction of the droplet with the surrounding medium and is dictated by the Reynolds number $\operatorname{Re}$ and the choice of $\sigma$. For $\sigma=0, U_{1}^{*}$ is zero but for $\sigma>0$ or $\sigma<0$ it indicates a migration in positive or negative $z$-direction, respectively. Interestingly, this refers to a plausible reversal of the migration direction from the migration induced by $U=U_{1}$ for $\sigma>0$. However, our aim is to analyse the droplet in its early unsettled phase. Therefore, in the present framework, all the swimming characteristics are analysed employing the migration velocity $U=U_{1}$.

\section{Second-order migration velocity}

In order to derive an expression for a migration velocity up to $O\left(R e^{2}\right)$, we stress that the net drag experienced by the droplet up to the same order must vanish across the surface. By employing the expression of the approximated streamfunction $\psi_{2}^{e}$ as in (3.25), we follow an equivalent formalism as summarized in $\S 4$. A lengthy and strenuous analysis shows that

$$
U=1+\operatorname{Re}(1+A \sigma)+\operatorname{Re}^{2}\left(11.460+B \sigma+C \sigma^{2}\right),
$$

where $A, B$, and $C$ are obtained as

$$
\left.\begin{array}{c}
A=0.134-0.064 \lambda+0.066 \lambda^{2}, \\
B=0.443-0.403 \lambda+0.995 \lambda^{2}, \\
C=0.0024+0.0035 \lambda-0.0085 \lambda^{2} .
\end{array}\right\}
$$

It may be noted that, throughout the analysis, by maintaining the $\lambda<1$ limit, we have considered terms up to $\lambda^{2}$. Likewise, we argue that the strengths of $\operatorname{Re}^{2} \lambda \sigma, \operatorname{Re}^{2} \lambda \sigma^{2}, \operatorname{Re}^{2} \lambda^{2} \sigma$ and $R e^{2} \lambda^{2} \sigma^{2}$ are less than unity in the present analysis; $U$ in (5.1) represents the real and coherent solution at $O\left(R e^{2}\right)$. In figure 7, a direct comparison of the swimming velocities as in (4.6) and (5.1) reveals that in the limit $R e<1$, as $U$ in (5.1) is viewed as quadratic power series of $R e$, it inherently predicts a higher upsurge of the migration velocity for both $\sigma>0$ and $\sigma<0$. Table 1 illustrates the quantitative increase $(U-1)$ of migration velocity with growing perturbations in Reynolds number. The conclusion from table 1 is that the difference between $O(R e)$ and $O\left(R e^{2}\right)$ is prominent in the range $R e \sim 10^{-1}$ at which the inertial effect is evident in the inner regime. Interestingly, we note that for a particular case when $C=0$ the contribution of the $\sigma^{2}$ is nullified in the migration velocity. Consequently, the disparity between $O(R e)$ and $O\left(R e^{2}\right)$ is curtailed reasonably. 


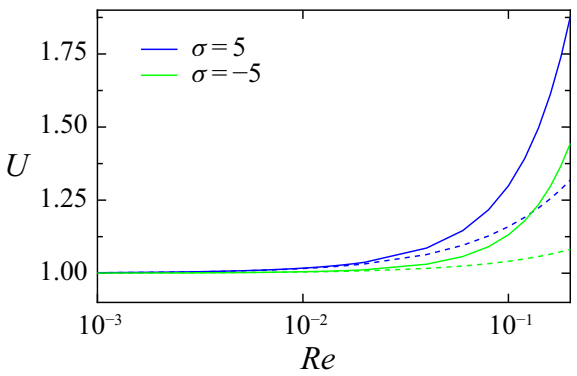

FIGURE 7. The comparison between the first-order $(O(R e))$ (dashed line) and second-order $\left(O\left(R^{2}\right)\right)$ (solid line) migration velocity with the Reynolds number for both $\sigma=5$ and $\sigma=-5$ at $\lambda=0.5$. As envisaged, the augmentations for both the cases are considerably higher for $O\left(R e^{2}\right)$.

\section{Conclusions}

In this work we have demonstrated the role of convective inertial forces of the external fluid medium on the self-propulsion of a viscous droplet with an inhomogeneous interfacial tension. We consider a generic axisymmetric framework for the inhomogeneous surface tension, and determine the exact analytical solution of the interior and exterior streamfunctions up to first $(O(R e))$ and an approximated second $\left(O\left(R e^{2}\right)\right)$ order solution in Reynolds number by utilizing the singular perturbation and matched asymptotic solution technique.

The discussion shows that, in the present model, the singular perturbation technique yields accurate migration velocity for the droplet under the assertion of low Reynolds number $(R e<1)$ and viscosity ratio $(\lambda<1)$ limits both at $O(R e)$ and $O\left(R e^{2}\right)$. It may be noted that the stress parameter $\sigma$ which is the ratio of the amplitudes of the first two modes of the stress field induced by the variable interfacial tension is comparable to $\beta$ for the solid squirmers. This distinguishes the swimming features of the active droplet and acts as a decisive parameter in governing the swimming characteristics of the droplet at finite $R e$.

In contrast to the solid squirmers, where the effect of inertia increases the speed of a pusher $(\beta<0)$ swimmer and decreases the speed of a puller $(\beta>0)$, in the case of swimming droplets the foreseen antisymmetry is broken. The finite inertial effect of the surrounding medium enables a systematic increase in the locomotion speed and swimming efficiency of the droplet for all $\sigma$ values. As was to be expected, at $O\left(R e^{2}\right)$, due to the quadratic coupling with the Reynolds number, the migration velocity exhibits a substantial increase for both $\sigma>0$ or $\sigma<0$ compared to $O(R e)$. However, the difference between $\sigma>0$ or $\sigma<0$ can be understood by considering how inertia perturbs the corresponding flow fields and is perceivable in the augmentation of the migration velocity.

Typically, a droplet with $\sigma<0$ is ineffective than its compliments due to its distinct swimming style. In this particular case, the stresslets generated by the droplet in the exterior medium intensify and the associated surface stress in the direction of migration increases at finite $R e$. This disadvantage is partially prevailed over by the presence of the counter-rotating vortices of the interior fluid towards the direction of migration. Interestingly, for $\sigma<0$, we identify that due to extended stresslets, the apparent extent of the swimmer increases at finite $R e$. This prompts an interplay among the inertial effect of the surrounding medium and the viscosity ratio of the droplet at higher $\lambda$ (in the limit $\lambda<1$ ), and subsequently the viscous power dissipation and the swimming efficiency differs from the monotonically decreasing and increasing Stokesian behaviour, 
respectively. Our findings also provide insights into an alternative illustration of the effect of inertia by visualizing the nonlinear drag function of the droplet in the phase space of the migration velocity. The linear stability analysis of the associated drag function at $O(R e)$ is to identify an asymptotically stable non-zero migration state for the droplet, which solely occurs due to the convective inertial forcing of the surrounding medium.

In summary, our approach is not restricted to a particular choice of external source that induces a variability in the surface tension, thus the above results can be applied to broader class of active droplets. Although the behaviours are manifested at the low viscosity ratio limit, the present framework probe deeper insights into the migration of a single swimming droplet at finite $R e$. The present results scale the migration velocity and relevant swimming characteristics of the droplet with the corresponding size and enhance the ability of the droplet to operate at higher flow rates. These are significant advantages for separation and filtration applications in micro-fluidic systems (Di Carlo 2009; Martel \& Toner 2014), and will stimulate experimental studies on artificial swimmers incorporating the inertial effect.

\section{Acknowledgements}

The second and third authors acknowledge the financial support from SERB, Government of India, project wide EMR/2016/000879. The authors report no conflict of interests.

\section{Declaration of interests}

The authors report no conflict of interest.

\section{Appendix A. Solution of streamfunction in the Oseen regime}

On employing the Goldstein transformation (Goldstein 1929), the leading-order contribution of $E_{\hat{r}}^{2} \Psi_{1}^{e}$ which necessarily satisfies $R e E_{\hat{r}}^{2} \Psi_{1}^{e} \sim O(1)$ in the stokes regime, can be written as (Proudman \& Pearson 1957; Khair \& Chisholm 2011)

$$
\begin{aligned}
E_{\hat{r}}^{2} \Psi_{1}^{e} & =\mathrm{e}^{\hat{r} \eta / 2}(\pi / 2)^{1 / 2} \mathrm{e}^{-\hat{r} / 2}\left(1+\frac{2}{\hat{r}}\right) \mathcal{A}_{1} Q_{1}(\eta) \\
& =\exp \left(-\frac{\hat{r}}{2}(1-\eta)\right)(\pi / 2)^{1 / 2}\left(1+\frac{2}{\hat{r}}\right) \mathcal{A}_{1} Q_{1}(\eta) .
\end{aligned}
$$

The vorticity $\left(\sim E^{2} \psi^{e}\right)$ generated by the swimmer in the inner regime primarily gets transported to the corresponding higher order in the Oseen regime (Khair \& Chisholm 2011). Subsequently, from the leading-order solution $\left(\psi_{0}^{e}\right)$ in the Stokes or inner regime, the associated contribution at $O(R e)$ is given by $E_{r}^{2} \psi_{0}^{e}=\left(\left(-2 B_{1}^{e}\right) / r\right) Q_{1}$. This leads to $\mathcal{A}_{1} \sim-B_{1}^{e}$. Consequently, from the results of Proudman \& Pearson (1957), the solution of $\Psi_{1}^{e}$ can be written as,

$$
\Psi_{1}^{e}=-B_{1}^{e}(1+\eta)\left(1-\exp \left(-\frac{\hat{r}(1-\eta)}{2}\right)\right) .
$$

\section{REFERENCES}

ARIYAPRAKAI, S. \& DUngan, S. R. 2008 Contribution of molecular pathways in the micellar solubilization of monodisperse emulsion droplets. Langmuir 24, 3061-3069. 
Balasubramaniam，R. \& Subramanian, R. S. 2000 The migration of a drop in a uniform temperature gradient at large Marangoni numbers. Phys. Fluids 12, 733.

BAtchelor, G. K. 1967 An Introduction to Fluid Dynamics. Cambridge University Press.

BÄUMLER, K. 2014 Simulation of single drops with variable interfacial tension. Doctoral thesis, Friedrich-Alexander-Universität Erlangen-Nürnberg (FAU).

BLAKE, J. R. 1971 Spherical envelope approach to ciliary propulsion. J. Fluid Mech. 46, 199-208.

BLYTH, M. G. \& PozRIKIDIS, C. 2014 Effect of inertia on the Marangoni instability of two-layer channel flow, part II: normal-mode analysis. J. Engng Maths 50, 329-341.

ČEJKovÁ, J., BAnno, T., Hanczyc, M. M. \& ŠTĚPÁnek, F. 2017 Droplets as liquid robots. Artif. Life 23, 528-549.

Chisholm, N. G., Legendre, D., Lauga, E. \& Khair, A. S. 2016 A squirmer across Reynolds numbers. J. Fluid Mech. 796, 233-256.

Clift, R., Grace, J. R. \& Weber, M. E. 1978 Bubbles, Drops, and Particles. Academic Press.

D’Ambrosio, D. \& Sinigaglia, F. 2003 Cell Migration in Inflammation and Immunity (ed. D. D'Ambrosio \& F. Sinigaglia). Springer.

Di CARLO, D. 2009 Inertial microfluidics. Lab Chip 9, 3038-3046.

Fadda, F., Gonnella, G., Lamura, A. \& Tiribocchi, A. 2017 Lattice Boltzmann study of chemically-driven self-propelled droplets. Eur. J. Phys. E 40, 112.

Furtado, K., Pooley, C. M. \& Yeomans, J. M. 2008 Lattice Boltzmann study of convective drop motion driven by nonlinear chemical kinetics. Phys. Rev. E 78, 046308.

GoldSTEIN, S. 1929 The steady flow of viscous fluid past a fixed spherical obstacle at small Reynolds numbers. Proc. R. Soc. Lond. A 123, 225-235.

Hamel, A., Fisch, C., Combettes, L., Dupuis-Williams, P. \& Baroud, C. N. 2011 Transitions between three swimming gaits in paramecium escape. Proc. Natl Acad. Sci. USA 108, 7290-7295.

HAPPEL, J. \& BRENNER, H. 1983 Low Reynolds Number Hydrodynamics. Springer.

Herminghaus, S., Maass, C. C., Kruger, C., Thutupalli, S., Goehring, L. \& Bahr, C. 2014 Interfacial mechanisms in active emulsions. Soft Matter 10, 7008-7022.

HsiaO, G. C. \& MACCAMY, R. C. 1982 Singular perturbations for the two-dimensional viscous flow problem. In Theory and Applications of Singular Perturbations, Lecture Notes in Mathematics, vol. 942. pp. 229-244.

HU, Y. T. \& LIPS, A. 2003 Estimating surfactant surface coverage and decomposing its effect on drop deformation. Phys. Rev. Lett. 91, 044501.

IZRI, Z., VAN DER Linden, M. N., Michelin, S. \& DAUCHOT, O. 2014 Self-propulsion of pure water droplets by spontaneous Marangoni-stress-driven motion. Phys. Rev. Lett. 113, 248302.

KAPLUN, S. 1957 Low Reynolds number flow past a circular cylinder. J. Fluid Mech. 6, 595-603.

Karbalaei, A., Kumar, R. \& Cho, H. J. 2016 Thermocapillarity in microfluidics-a review. Micromachines 77, 1.

Khair, A. S. \& Chisholm, N. G. 2011 Expansions at small Reynolds numbers for the locomotion of a spherical squirmer. Phys. Fluids 24, 101902.

Kim, S. \& KARRILA, S. J. 2005 Microhydrodynamics: Principles and Selected Applications. Dover Publications.

Kitahata, H., Yoshinaga, N., Nagai, K. H. \& Sumino, Y. 2011 Spontaneous motion of a droplet coupled with a chemical wave. Phys. Rev. E 84, 015101.

KreE, R., BURADA, P. S. \& Zippelius, A. 2017 From active stresses and forces to self-propulsion of droplets. J. Fluid Mech. 821, 595-623.

LAmorgese, A. G. \& MAURI, R. 2011 Liquid mixture convection during phase separation in a temperature gradient. Phys. Fluids 23, 034102.

LAmorgese, A. G. \& MAURI, R. 2017 Phase-field modeling of mixing/demixing of regular binary mixtures with a composition-dependent viscosity. J. Appl. Phys. 121, 134302.

Lauga, E. \& Davis, A. M. J. 2012 Viscous Marangoni propulsion. J. Fluid Mech. 705, 120-133.

Lauga, E. \& Powers, T. R. 2009 The hydrodynamics of swimming microorganisms. Rep. Prog. Phys. 72, 096601.

LeVAn, M. D. 1981 Motion of a droplet with a newtonian interface. J. Colloid Interface Sci. 83, 11-17. 
Li, M., Brinkmann, M., Pagonabarraga, I., Seemann, R. \& Fleury, J. B. 2018 Spatiotemporal control of cargo delivery performed by programmable self-propelled janus droplets. Commun. Phys. $1,23$.

LI, X. J. \& MAO, Z. S. 2001 The effect of surfactant on the motion of a buoyancy-driven drop at intermediate Reynolds numbers: a numerical approach. J. Colloid Interface Sci. 240, 307-322.

LighthilL, M. J. 1952 On the squirming motion of nearly spherical deformable bodies through liquids at very small Reynolds numbers. Commun. Pure Appl. Maths 5, 109.

Majss, C. C., Krúger, C., Herminghaus, S. \& Bahr, C. 2016 Swimming droplets. Annu. Rev. Condens. Matter Phys. 7, 171-193.

Martel, J. M. \& Toner, M. 2014 Inertial focusing in microfluidics. Annu. Rev. Biomed. Engng 16, 371-396.

MASON, D. P. \& Moremedi, G. M. 2011 Effects of non-uniform interfacial tension in small Reynolds number flow past a spherical liquid drop. Pramana 77, 493-507.

Masoud, H. \& Stone, H. A. 2014 A reciprocal theorem for Marangoni propulsion. J. Fluid Mech. 741, R4.

Masoud, H. \& StOne, H. A. 2019 The reciprocal theorem in fluid dynamics and transport phenomena. J. Fluid Mech. 879, P1.

Mozorov, M. \& Michelin, M. 2019 Self-propulsion near the onset of Marangoni instability of deformable active droplets. J. Fluid Mech. 860, 711-738.

Oseen, C. W. 1910 On the stability problem in hydrodynamics. Ark. Mat. Astron. Fys. 6, 29.

Pena, A. A. \& Miller, C. A. 2006 Solubilization rates of oils in surfactant solutions and their relationship to mass transport in emulsions. Adv. Colloid Interface Sci. 123, 241-260.

Proudman, I. \& Pearson, J. R. A. 1957 Expansions at small Reynolds numbers for the flow past a sphere and a circular cylinder. J. Fluid Mech. 2, 237-262.

Purcell, E. M. 1977 Life at low Reynolds number. Am. J. Phys. 45, 3.

REYNOLDS, A. J. 1965 The swimming of minute organisms. J. Fluid Mech. 23, 241-260.

Sadhal, S., Ayyaswamy, P. \& Chung, J. 1997 Transport Phenomena with Drops and Bubbles. Springer.

Saintillan, D. \& Shelley, M. J. 2015 Theory of active suspensions. In Complex Fluids in Biological Systems (ed. S. Spagnolie). Springer.

SAVILLE, D. A. 1973 The effects of interfacial tension gradients on the motion of drops and bubbles. Chem. Engng J. 5, 251-259.

Schmitt, M. \& StARK, H. 2013 Swimming active droplet: a theoretical analysis. Eur. Phys. Lett. 101, 44008.

SCHMITT, M. \& STARK, H. 2016 a Active Brownian motion of emulsion droplets: coarsening dynamics at the interface and rotational diffusion. Eur. J. Phys. E 39, 80.

SCHMitT, M. \& StARK, H. 2016b Marangoni flow at droplet interfaces: three-dimensional solution and applications. Phys. Fluids 28, 012106.

SCRIVEN, L. E. \& STERnLing, C. V. 1960 The Marangoni effects. Nature 187, 186-188.

Seemann, R., Fleury, J. B. \& MaAss, C. C. 2016 Self-propelled droplets. Eur. Phys. J. Spec. Top. 225, 2227-2240.

SERIC, I., AfKhAMI, S. \& Kondic, L. 2018 Direct numerical simulation of variable surface tension flows using a volume-of-fluid method. J. Comput. Phys. 352, 615-636.

Shardt, O., Masoud, H. M. \& Stone, H. A. 2016 Oscillatory Marangoni flows with inertia. J. Fluid Mech. 803, 94-118.

Stone, H. A. \& Leal, L. G. 1990 The effects of surfactants on drop deformation and breakup. J. Fluid Mech. 220, 161-186.

Stone, H. A. \& Samuel, T. D. 1996 Propulsion of microorganisms by surface distortions. Phys. Rev. Lett. 106, 4102.

STRICKER, L. 2017 Numerical simulation of artificial microswimmers driven by Marangoni flow. $J$. Comput. Phys 347, 467-489.

Subramanian, R. S. 1992 Thermocapillary Motion of Bubbles and Drops. Springer.

TAYlor, T. D. \& ACRIVOS, A. 1964 On the deformation and drag of a falling viscous drop at low Reynolds number. J. Fluid Mech. 18, 466-476. 
Thutupalli, S. \& Herminghaus, S. 2011 Tuning active emulsion dynamics via surfactants and topology. Eur. J. Phys. E 36, 91.

VAn Dy Ke, M. 1975 Perturbation Methods in Fluid Mechanics. Academic Press.

WANG, S. \& ARdeKANI, A. 2011 Inertial squirmer. Phys. Fluids 24, 101902.

Weber, C. A, Zwicker, D., JÜlicher, F. \& Lee, C. F. 2019 Physics of active emulsions. Rep. Prog. Phys. 83, 064601.

WhiteHEAD, A. N. 1889 Second approximations to viscous fluid motion. Q. J. Maths 23, 143-152.

Wu, Z., Willing, B., BJerketorP, J., JANSSON, J. K. \& HJORT, K. 2009 Soft inertial microfluidics for high throughput separation of bacteria from human blood cells. Lab Chip 9, 1193-1199.

YoshinaGa, N. 2017 Simple models of self-propelled colloids and liquid drops: from individual motion to collective behaviors. J. Phys. Soc. Japan 86, 101009.

Zhang, L. U., Subramanian, R. S. \& Balasubramaniam, R. 2001 Motion of a drop in a vertical temperature gradient at small Marangoni number-the critical role of inertia. J. Fluid Mech. 448, 199-211. 